\title{
Three-dimensional effects of coherent synchrotron radiation by electrons in a bunch compressor
}

\author{
Yunhai Cai and Yuantao Ding \\ SLAC National Accelerator Laboratory, 2575 Sand Hill Road, Menlo Park, California 94205, USA
}

(Received 23 May 2019; published 9 January 2020)

\begin{abstract}
We develop a three-dimensional theory of coherent synchrotron radiation in steady-state. Starting from the Hamiltonian in the Courant-Synder theory in a curvilinear coordinate system, we find that the transverse force is essentially the Lorentz force with an additional term: $-e \phi / \rho$ in the bending plane, where $\phi$ is the potential and $\rho$ the bending radius. The potential term partially cancels the centrifugal force, resulting in a residual wakefield: $-\Lambda \delta(z) / \rho$, where, $2 \leq \Lambda \leq 4$ and $z$ is the longitudinal coordinate. The measurement in the bunch compressor supports the sign predicted by the theory but with a smaller value.
\end{abstract}

DOI: 10.1103/PhysRevAccelBeams.23.014402

\section{INTRODUCTION}

Coherent synchrotron radiation (CSR) in bunch compressors places an important limit for advancing the brightness of the electron beam in free electron laser facilities and linear colliders. It degrades the beam quality, causing microbunching $[1,2]$ and increasing energy spread and emittance. It is well known that the dominant effect is due to the longitudinal wakefield [3,4], including the transient effects $[5,6]$. The longitudinal wake was implemented in ELEGANT [7], which is routinely used for the design of bunch compressors. The simulations are largely in good agreement with the experimental observations [8]. Despite its success, as a theory, it is not quite complete because of the lack of an explanation of why the transverse force can be ignored.

In the transverse planes, the centrifugal force was first proposed by Talman [9]. The force was carefully analyzed by Lee [10], who showed that it is magically cancelled by the potential term due to the energy change within a coasting beam. For a bunched beam, Derbenev and Shiltsev [11] showed a similar cancellation but with a residual centripetal wake as given in the abstract with $\Lambda=2$. Another derivation was given recently by Stupakov [12]. Despite these theoretical studies, the cancellation of the centrifugal force remains as an unsettled and controversial topic $[13,14]$ partially because of the lack of a derivation of the potential term based on the standard theory of particle accelerators.

Published by the American Physical Society under the terms of the Creative Commons Attribution 4.0 International license. Further distribution of this work must maintain attribution to the author(s) and the published article's title, journal citation, and DOI.
There were many efforts in simulations by numerically solving electromagnetic fields $[15,16]$, tracking particles with particle-in-cell [17] or distribution with the Vlasov method [18]. Among them, the most common code is CSRtrack [19] for two- or three-dimensional effects. The code was benchmarked against the measurements with a reasonable agreement. Due to the limitation of computing speed, these codes are hardly ever used for the design of a bunch compressor when a large scan of the parameter space is often required. Similarly, the codes are not systematically compared to the theory.

Our primary goal in this paper is to extend our previous work of a two-dimensional theory [20] to a threedimensional one and to lay a solid foundation for further advances toward a complete theory of CSR. As a secondary goal, we want to lay a groundwork for developing a robust code for simulation.

\section{ELECTRIC AND MAGNETIC FIELDS}

Now we consider the relativistic effects in computing the fields. For simplicity, we assume that all particles have no vertical velocities and therefore are moving on circular orbits. In particular, when a charged particle is moving in a circular orbit from point $\mathrm{A}$ to $\mathrm{C}$ as illustrated in Fig. 1, it generates electromagnetic fields, which can be expressed by the Lienard-Wiechert formula [21],

$$
\begin{aligned}
\vec{E}=e\left[\frac{\hat{n}-\vec{\beta}}{\gamma^{2}(1-\vec{\beta} \cdot \hat{n})^{3} R^{2}}\right]_{\mathrm{ret}} & +\frac{e}{c}\left[\frac{\hat{n} \times\{(\hat{n}-\vec{\beta}) \times \dot{\vec{\beta}}\}}{(1-\vec{\beta} \cdot \hat{n})^{3} R}\right]_{\mathrm{ret}}, \\
\vec{B} & =\hat{n} \times \vec{E},
\end{aligned}
$$

where $\hat{n}=\vec{R} / R$ and $\vec{R}$ is the vector from the point A, where the source particle is at the retarded time $t^{\prime}$ and, to D the observation point at the time $t$. Moreover, the point $\mathrm{B}$ is 


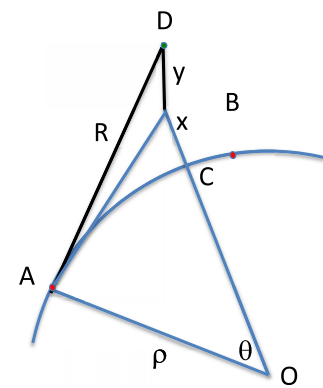

FIG. 1. A source particle at the point A (in red color) moves along a circular orbit and radiates electromagnetic fields that reach a test particle at the point $\mathrm{D}$ (green). The black colored lines are out of the orbiting plane.

where the source particle at the same time $t$. The retarded time is given by,

$$
t^{\prime}=t-\frac{R}{c}
$$

The associated angle $\theta$ is the retarded angle.

\section{RETARDED CONDITION}

Since the expressions of the fields are subject to the retarded condition in Eq. (3), we need to solve the equation in terms of the position of the source particle at the moment $t$. From Fig. 1, we can see that the arc length between point $\mathrm{C}$ and $\mathrm{B}$ is,

$$
\ell=v\left(t-t^{\prime}\right)-\left(s-s^{\prime}\right),
$$

which can be identified as the longitudinal position of the source particle relative to the test particle at the moment $t$, namely $\ell=z^{\prime}-z$. Using the retarded condition in Eq. (3), we can rewrite Eq. (4) in terms of $\alpha$,

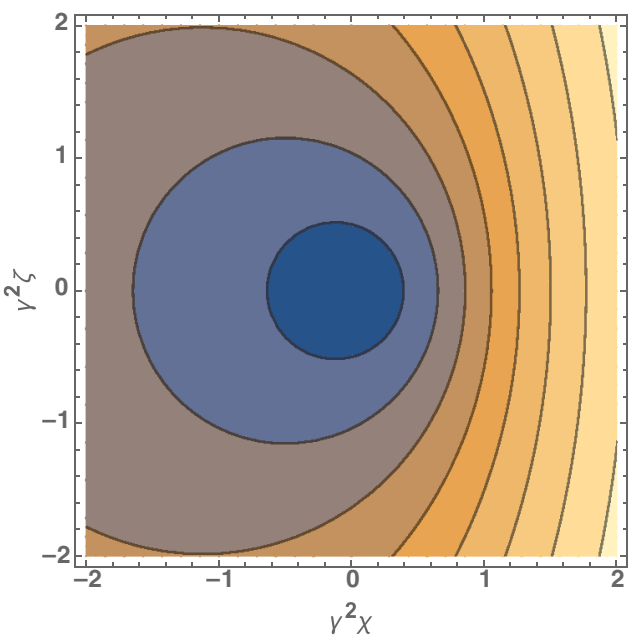

$$
\xi=\alpha-\frac{\beta}{2} \sqrt{\chi^{2}+\zeta^{2}+4(1+\chi) \sin ^{2} \alpha},
$$

where $\chi=x / \rho, \zeta=y / \rho, \xi=-\ell / 2 \rho$, and $\alpha=\theta / 2$ is a half of the retarded angle. Given $\chi, \zeta$, and $\xi$, the angle $\alpha$ can be found with a Newton searching using Eq. (5).

Expanding up to fourth order of $\alpha$, Eq. (5) can be rewritten as,

$$
\begin{gathered}
\alpha^{4}+\frac{3\left(1-\beta^{2}-\beta^{2} \chi\right)}{\beta^{2}(1+\chi)} \alpha^{2}-\frac{6 \xi}{\beta^{2}(1+\chi)} \alpha \\
+\frac{3\left(4 \xi^{2}-\beta^{2} \chi^{2}-\beta^{2} \zeta^{2}\right)}{4 \beta^{2}(1+\chi)}=0 .
\end{gathered}
$$

The solution of this quartic equation is given in Appendix A. A comparison between the numerical and analytical solutions in the plane of a very small $\xi$ is carried out and shown in Fig. 2. The difference $\gamma \Delta \alpha$ is at the level of $10^{-6}$. A similar comparison in the plane of $\zeta=0$ has been published in the previous paper. The agreement is so good that we will continue to use the analytic solution for its computational efficiency in this paper.

\section{COHERENT SYNCHROTRON RADIATION}

The first term in Eq. (1) is the space-charge force. Because of the suppression factor of $1 / \gamma^{2}$, it is not important for ultrarelativistic electrons. In this paper, we will briefly discuss the space-charge term in Appendix B and instead focus on the second term that is associated with acceleration, defined as $\dot{\vec{\beta}}=d \vec{\beta}\left(t^{\prime}\right) / d t^{\prime}$. With the radius $\rho$ of the circle, we have $\dot{\beta}=c \beta^{2} / \rho$. Using the acceleration and trigonometry, it is straightforward to compute the radiative electromagnetic fields. Their components can be written as,

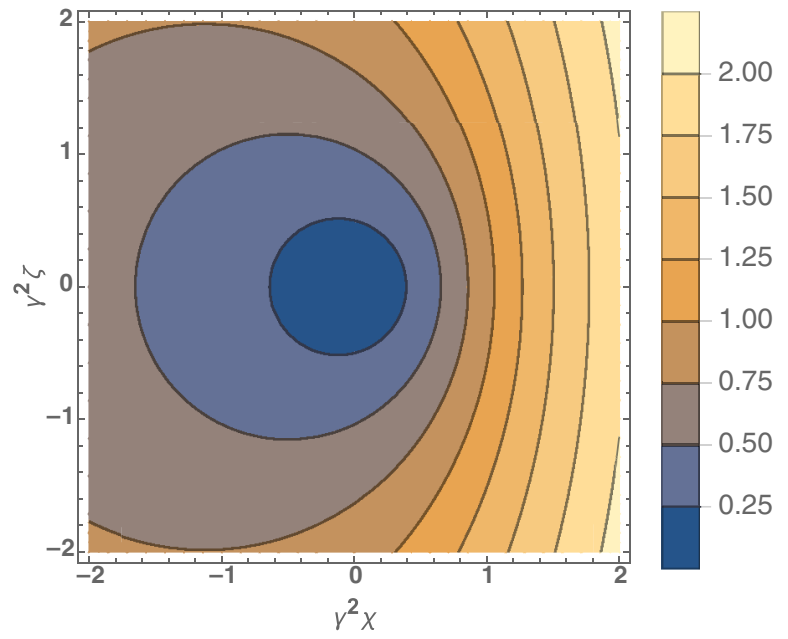

FIG. 2. Comparison of the numerical (left) and analytical (right) solutions of $\gamma \alpha$ as a function of $\gamma^{2} \chi$ and $\gamma^{2} \zeta$ with $\xi=1 / 300 \gamma^{3}$ and $\gamma=500$. 


$$
\begin{gathered}
E_{x}=\frac{e \beta^{2}\left\{\sin 2 \alpha[(1+\chi) \sin 2 \alpha-\beta \kappa]+\zeta^{2} \cos 2 \alpha\right\}}{\rho^{2}[\kappa-\beta(1+\chi) \sin 2 \alpha]^{3}} \\
E_{y}=\frac{e \beta^{2} \zeta[1-(1+\chi) \cos 2 \alpha]}{\rho^{2}[\kappa-\beta(1+\chi) \sin 2 \alpha]^{3}}, \\
E_{s}=\frac{e \beta^{2}\left\{[\cos 2 \alpha-(1+\chi)][(1+\chi) \sin 2 \alpha-\beta \kappa]-\zeta^{2} \sin 2 \alpha\right\}}{\rho^{2}[\kappa-\beta(1+\chi) \sin 2 \alpha]^{3}}, \\
B_{x}=-\frac{e \beta^{2} \zeta\{\kappa \sin 2 \alpha+\beta[\cos 2 \alpha-(1+\chi)]\}}{\rho^{2}[\kappa-\beta(1+\chi) \sin 2 \alpha]^{3}} \\
B_{y}=\frac{e \beta^{2}\left\{\kappa[(1+\chi) \sin 2 \alpha-\beta \kappa]+\zeta^{2} \beta\right\}}{\rho^{2}[\kappa-\beta(1+\chi) \sin 2 \alpha]^{3}} \\
B_{s}=\frac{e \beta^{2} \zeta[\beta \sin 2 \alpha-\kappa \cos 2 \alpha]}{\rho^{2}[\kappa-\beta(1+\chi) \sin 2 \alpha]^{3}}
\end{gathered}
$$

where $\kappa=R / \rho$ is given explicitly,

$$
\kappa=\sqrt{\chi^{2}+\zeta^{2}+4(1+\chi) \sin ^{2} \alpha} .
$$

There are many equivalent expressions of the fields given the relations in Eq. (13). Here, we have chosen the ones with simple factors, especially in the denominator. Otherwise, the numerical noise will overwhelm the fields near the singularity. Note that $E_{y}, B_{x}$, and $B_{s}$ are an order of $\zeta$ smaller than the other three components. Moreover, in the bending plane $\zeta=0$, they vanish and the other nonzero components reduce to the ones in the two-dimensional theory [20].

\section{TRANSVERSE FORCE}

Given the electromagnetic fields, how do they affect the motion of the particles? The equation of the motion can be derived from the Hamiltonian in the Courant-Synder theory [22] in a curvilinear coordinate system. A derivation can be found [20] and they are written as,

$$
\begin{gathered}
x^{\prime \prime}+\frac{x}{\rho^{2}}=\frac{\delta}{\rho}+\frac{e}{c p_{0} \beta_{s}}\left[E_{x}+\beta_{y} B_{s}-\beta_{s}\left(1+\frac{x}{\rho}\right) B_{y}\right]-\frac{e \phi}{c p_{0} \beta \rho}, \\
y^{\prime \prime}=\frac{e}{c p_{0} \beta_{s}}\left[E_{y}+\beta_{s}\left(1+\frac{x}{\rho}\right) B_{x}-\beta_{x} B_{s}\right], \\
\delta=\frac{e}{c p_{0} \beta_{s} \beta} \vec{\beta} \cdot \vec{E} .
\end{gathered}
$$

where $\vec{\beta}=\vec{v} / c$ is the velocity normalized to the speed of light and the derivative is with respect to the arc length. The transverse motion is largely driven by the electric and magnetic fields as expected, provided that the Lorentz force is modified with extra terms that are proportional to $x / \rho$. The importance of these terms has been emphasized in our previous paper [20].

More importantly, there is a potential term in the horizontal plane, which was first proposed by Lee [10] to cancel the centrifugal force found by Talman [9]. Often it is introduced heuristically by an argument concerning the energy change due to the potential. Here we have derived it from the product of the energy and the potential in the square root. Note that this term was neglected in our previous paper. In this paper, we will study the consequences of the potential term.

Applying the paraxial approximation: $\beta_{x} \approx 0, \beta_{y} \approx 0$, and $\beta_{s} \approx \beta$, and reading from Eq. (14) and Eqs. (7)-(12), the transverse force is given by,

$$
\begin{gathered}
F_{x}=\frac{e^{2} \beta^{2}\left\{[\sin 2 \alpha-\beta(1+\chi) \kappa][(1+\chi) \sin 2 \alpha-\beta \kappa]+\zeta^{2}\left[\cos 2 \alpha-(1+\chi) \beta^{2}\right]\right\}}{\rho^{2}[\kappa-\beta(1+\chi) \sin 2 \alpha]^{3}}, \\
F_{\phi}=\frac{e^{2}}{\rho^{2}[\kappa-\beta(1+\chi) \sin 2 \alpha]}, \\
F_{y}=\frac{e^{2} \beta^{2} \zeta\{1-(1+\chi) \cos 2 \alpha-\beta(1+\chi)[\kappa \sin 2 \alpha+\beta \cos 2 \alpha-\beta(1+\chi)]\}}{\rho^{2}[\kappa-\beta(1+\chi) \sin 2 \alpha]^{3}},
\end{gathered}
$$

where $F_{\phi}=e \phi / \rho$ has been computed using the Lienard-Wiechert potential [21],

$$
\phi=\frac{e}{(1-\vec{\beta} \cdot \hat{n}) R} .
$$

As a result, the effective force in the horizontal plane is given by $\hat{F}_{x}=F_{x}-F_{\phi}$. 

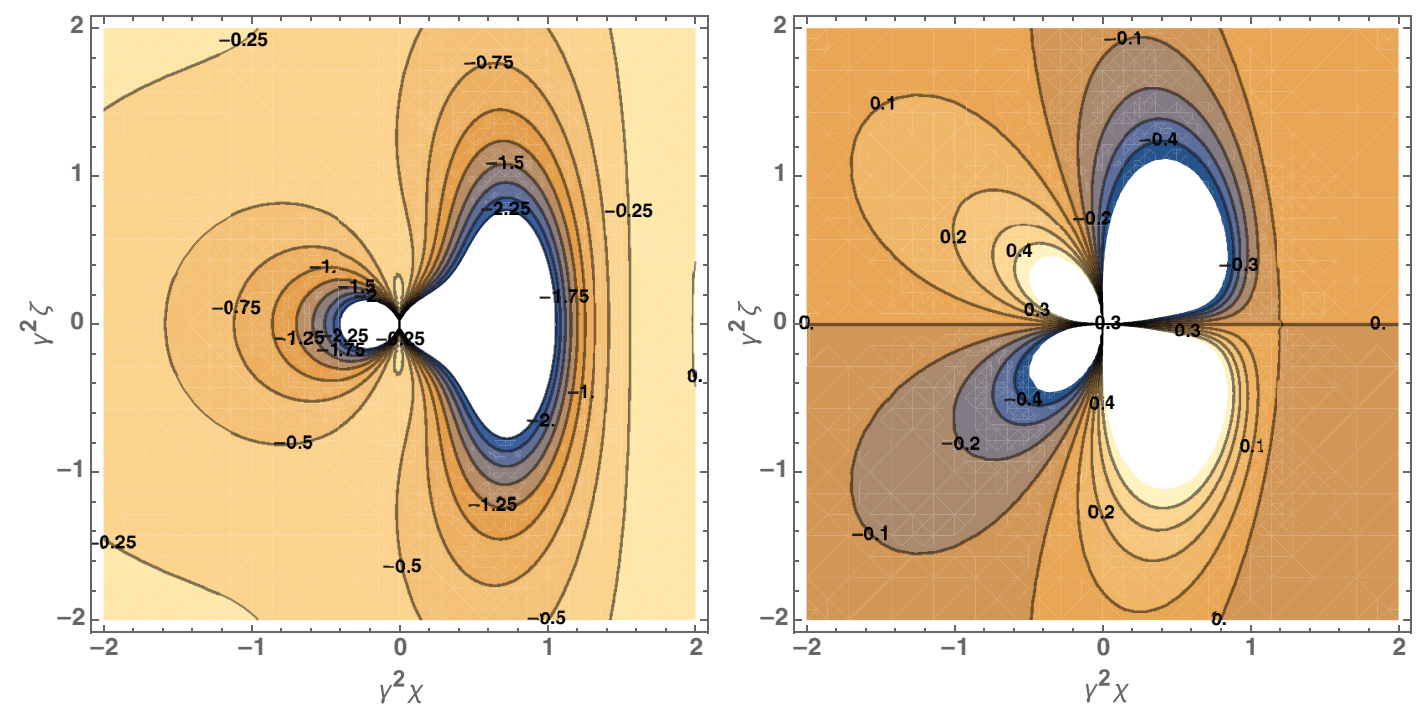

FIG. 3. The horizontal effective force $\left(F_{x}-F_{\phi}\right) \rho^{2} / e^{2} \gamma^{3}$ (left) and the vertical force $F_{y} \rho^{2} / e^{2} \gamma^{3}$ (right) as a function of $\gamma^{2} \chi$ and $\gamma^{2} \zeta$ at a longitudinal position of $\xi=1 / 300 \gamma^{3}$ with $\gamma=500$.

Knowing $\alpha$, we compute and plot $\hat{F}_{x}$ and $F_{y}$ in Fig. 3 respectively. The scalings of the plots are chosen according to their approximated invariance with respect to $\gamma$. The singularity and complicity of the radiation force is clearly seen in the figure. In the horizontal plane, we see mostly dipole fields while in the vertical plane, sextupole fields. Because of the singular features as two particles are approaching each other, it is difficult to use $F_{x}-F_{\phi}$, or $F_{y}$ as Green's functions for a bunch distribution.

\section{POTENTIALS}

Here we continue on dealing with the singularity. A method to avoid the singularity in the Green's functions is integration by parts. It requires us to know the integral of the Green's function. Differentiating the retarded condition in Eq. (5), we have

$$
d \xi=\left(1-\frac{\beta(1+\chi) \sin 2 \alpha}{\sqrt{\chi^{2}+\zeta^{2}+4(1+\chi) \sin ^{2} \alpha}}\right) d \alpha .
$$

Combining it with the longitudinal electric field $E_{s}$ in Eq. (9), we find

$$
E_{s} d \xi=\frac{e \beta^{2}\left\{[\cos 2 \alpha-(1+\chi)][(1+\chi) \sin 2 \alpha-\beta \kappa]-\zeta^{2} \sin 2 \alpha\right\}}{\rho^{2} \kappa[\kappa-\beta(1+\chi) \sin 2 \alpha]^{2}} d \alpha=d\left\{\frac{e \beta^{2}\left(\cos 2 \alpha-\frac{1}{1+\chi}\right)}{2 \rho^{2}[\kappa-\beta(1+\chi) \sin 2 \alpha]}\right\} .
$$

This relation naturally leads to a longitudinal potential,

$$
\Psi_{s}(\chi, \zeta, \xi)=\frac{e \beta^{2}\left(\cos 2 \alpha-\frac{1}{1+\chi}\right)}{2 \rho^{2}[\kappa-\beta(1+\chi) \sin 2 \alpha]},
$$

and $E_{s}=d \Psi_{s} / d \xi$.

Similarly, for the transverse force, we have $F_{x}=e d \Psi_{x} / d \xi$ and $F_{\phi}=e d \Psi_{\phi} / d \xi$ with the horizontal potentials,

$$
\begin{aligned}
\Psi_{x}(\chi, \zeta, \xi)= & \frac{e \beta^{2}}{2 \rho^{2}}\left\{\frac{\left(2+2 \chi+\chi^{2}\right) F\left(\alpha, \frac{-4(1+\chi)}{\chi^{2}+\zeta^{2}}\right)}{(1+\chi) \sqrt{\chi^{2}+\zeta^{2}}}-\frac{\left.\left[\chi^{2}(2+\chi)^{2}\right]+\zeta^{2}\left(2+2 \chi+\chi^{2}\right)\right] E\left(\alpha, \frac{-4(1+\chi)}{\chi^{2}+\zeta^{2}}\right)}{(1+\chi)\left[\zeta^{2}+(2+\chi)^{2}\right] \sqrt{\chi^{2}+\zeta^{2}}}\right. \\
& +\frac{\kappa^{2}-2 \beta^{2}(1+\chi)^{2}+\beta^{2}(1+\chi)\left(2+2 \chi+\chi^{2}\right) \cos 2 \alpha}{\beta(1+\chi)\left[\kappa^{2}-\beta^{2}(1+\chi)^{2} \sin ^{2} 2 \alpha\right]}+\frac{\kappa\left[\zeta^{4}-\chi^{2}(2+\chi)^{2}-2 \beta^{2} \zeta^{2}(1+\chi)^{2}\right] \sin 2 \alpha}{\left(\chi^{2}+\zeta^{2}\right)\left[\zeta^{2}+(2+\chi)^{2}\right]\left[\kappa^{2}-\beta^{2}(1+\chi)^{2} \sin ^{2} 2 \alpha\right]} \\
& \left.+\frac{\kappa \beta^{2}(1+\chi)\left[\chi^{2}(2+\chi)^{2}+\zeta^{2}\left(2+2 \chi+\chi^{2}\right)\right] \sin 2 \alpha \cos 2 \alpha}{\left(\chi^{2}+\zeta^{2}\right)\left[\zeta^{2}+(2+\chi)^{2}\right]\left[\kappa^{2}-\beta^{2}(1+\chi)^{2} \sin ^{2} 2 \alpha\right]}\right\} \\
\Psi_{\phi}(\chi, \zeta, \xi)= & \frac{e}{\rho^{2} \sqrt{\chi^{2}+\zeta^{2}}} F\left(\alpha, \frac{-4(1+\chi)}{\chi^{2}+\zeta^{2}}\right)
\end{aligned}
$$



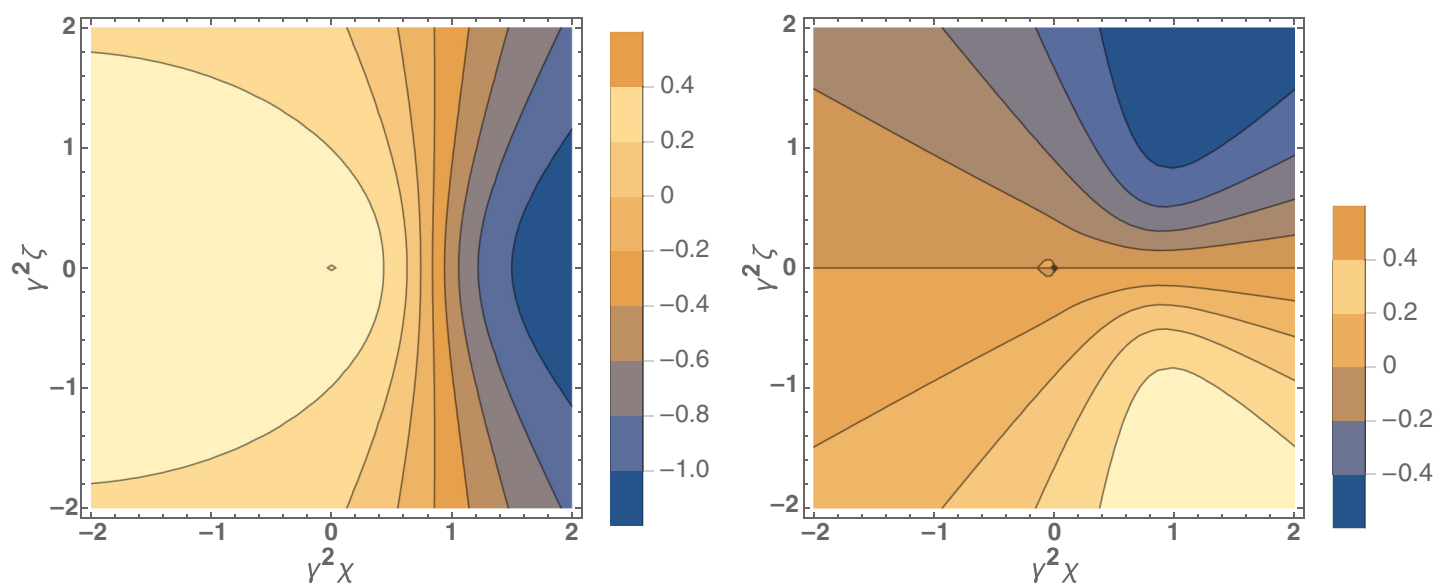

FIG. 4. The horizontal effective potential $\left(\Psi_{x}-\Psi_{\phi}\right) \rho^{2} / e$ (left) and the vertical potential $\Psi_{y} \rho^{2} / e$ (right) as a function of $\gamma^{2} \chi$ and $\gamma^{2} \zeta$ at a longitudinal position of $\xi=1 / 300 \gamma^{3}$ with $\gamma=500$.

where $F(\alpha, k)$ and $E(\alpha, k)$ are the incomplete elliptic integrals of the first and second kind respectively. Note that the curvature term in the transverse force in Eq. (17) is necessary to obtain the expression. In the vertical plane, the potential is given by,

$$
\begin{aligned}
\Psi_{y}(\chi, \zeta, \xi)= & \frac{e \beta^{2} \zeta}{2 \rho^{2}}\left\{\frac{F\left(\alpha, \frac{-4(1+\chi)}{\chi^{2}+\zeta^{2}}\right)}{\sqrt{\chi^{2}+\zeta^{2}}}-\frac{\left[\chi(2+\chi)+\zeta^{2}\right] E\left(\alpha, \frac{-4(1+\chi)}{\chi^{2}+\zeta^{2}}\right)}{\left[\zeta^{2}+(2+\chi)^{2}\right] \sqrt{\chi^{2}+\zeta^{2}}}-\frac{\beta[1-(1+\chi) \cos 2 \alpha]}{\left[\kappa^{2}-\beta^{2}(1+\chi)^{2} \sin ^{2} 2 \alpha\right]}\right. \\
& +\frac{\kappa(1+\chi)\left[-\left(2+\beta^{2}\right) \zeta^{2}+\left(-2+\beta^{2}\right) \chi(2+\chi)\right] \sin 2 \alpha}{\left[\zeta^{4}+\chi^{2}(2+\chi)^{2}+2 \zeta^{2}\left(2+2 \chi+\chi^{2}\right)\right]\left[\kappa^{2}-\beta^{2}(1+\chi)^{2} \sin ^{2} 2 \alpha\right]} \\
& \left.+\frac{\kappa \beta^{2}(1+\chi)^{2}\left[\zeta^{2}+\chi(2+\chi)\right] \sin 2 \alpha \cos 2 \alpha}{\left[\zeta^{4}+\chi^{2}(2+\chi)^{2}+2 \zeta^{2}\left(2+2 \chi+\chi^{2}\right)\right]\left[\kappa^{2}-\beta^{2}(1+\chi)^{2} \sin ^{2} 2 \alpha\right]}\right\},
\end{aligned}
$$

where $F_{y}=e d \Psi_{y} / d \xi$. Note that $\Psi_{y}$ is much less divergent at the singularity and smaller than $\Psi_{x}$ because of the suppression factor $\zeta$.

Similarly, we compute and plot the effective potential in the horizontal plane $\hat{\Psi}_{x}=\Psi_{x}-\Psi_{\phi}$ and the vertical potential $\Psi_{y}$ in Fig. 4 respectively. The singularity seen in the radiation forces in Fig. 3 are clearly removed. In particular, the "logarithmic" singularity in $\Psi_{x}$ is essentially cancelled by $\Psi_{\phi}$ in the effective potential. As a result, the potentials $\hat{\Psi}_{x}$ and $\Psi_{y}$ can be used as Green's functions for a derivative of bunch distribution.

\section{BUNCH WAKES}

Given the potentials in Eqs. (23)-(25), and the equations of the motion in Eqs. (14) and (16), we derive a change of the momentum deviation $\delta$ and a kick to $x^{\prime}$ and $y^{\prime}$ by the bunch wakefields

$$
\begin{aligned}
& \delta=\frac{r_{e} N_{b}}{\gamma} W_{s}(\chi, \zeta, z), \\
& x^{\prime \prime}=\frac{r_{e} N_{b}}{\gamma} \hat{W}_{x}(\chi, \zeta, z),
\end{aligned}
$$

$$
y^{\prime \prime}=\frac{r_{e} N_{b}}{\gamma} W_{y}(\chi, \zeta, z)
$$

where $r_{e}$ is the classical electron radius, $N_{b}$ the bunch population, and the wakes are

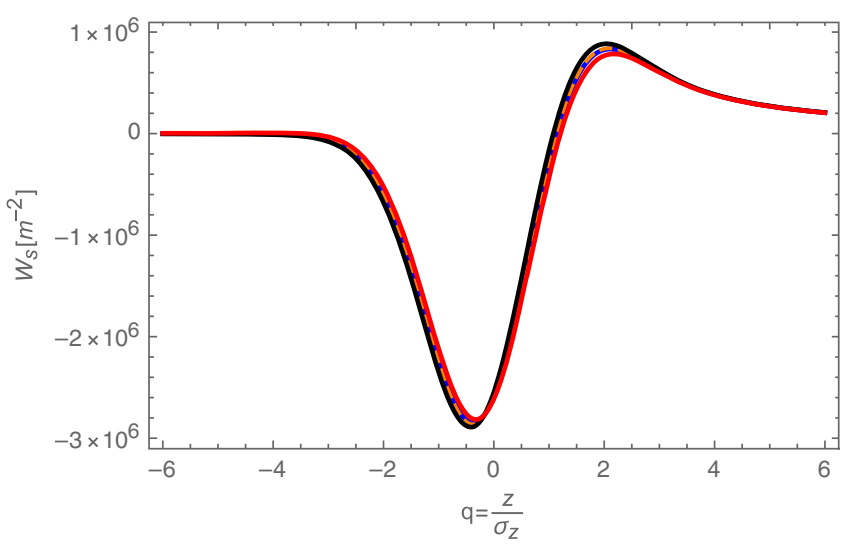

FIG. 5. The longitudinal wakes at various horizontal positions: $\chi=-\sigma_{x} / \rho$ (in black color), 0 (blue), and $\sigma_{x} / \rho$ (red) in a comparison to the one-dimensional theory (dashed orange). 


$$
\begin{aligned}
& W_{s}(\chi, \zeta, z)=\iiint \mathcal{Y}_{s}\left(\chi-\chi^{\prime}, \zeta-\zeta^{\prime}, \frac{z-z^{\prime}}{2 \rho}\right) \frac{\partial \lambda_{b}\left(\chi^{\prime}, \zeta^{\prime}, z^{\prime}\right)}{\partial z^{\prime}} d \chi^{\prime} d \zeta^{\prime} d z^{\prime}, \\
& \hat{W}_{x}(\chi, \zeta, z)=\iiint \hat{\mathcal{Y}}_{x}\left(\chi-\chi^{\prime}, \zeta-\zeta^{\prime}, \frac{z-z^{\prime}}{2 \rho}\right) \frac{\partial \lambda_{b}\left(\chi^{\prime}, \zeta^{\prime}, z^{\prime}\right)}{\partial z^{\prime}} d \chi^{\prime} d \zeta^{\prime} d z^{\prime}, \\
& W_{y}(\chi, \zeta, z)=\iiint \mathcal{Y}_{y}\left(\chi-\chi^{\prime}, \zeta-\zeta^{\prime}, \frac{z-z^{\prime}}{2 \rho}\right) \frac{\partial \lambda_{b}\left(\chi^{\prime}, \zeta^{\prime}, z^{\prime}\right)}{\partial z^{\prime}} d \chi^{\prime} d \zeta^{\prime} d z^{\prime},
\end{aligned}
$$

with $\mathcal{Y}_{s}=2 \rho \Psi_{s} / e, \hat{\mathcal{Y}}_{x}=2 \rho \hat{\Psi}_{x} / e, \mathcal{Y}_{y}=2 \rho \Psi_{y} / e$, and $\lambda_{b}$ an arbitrary distribution that is normalized to $\iint \lambda_{b} d \chi d \zeta d z=1$. The bunch wakes should be computed at progressive locations along a beamline and used to update the changes of $\delta, x^{\prime}$ and $y^{\prime}$ respectively.

As a numerical example, we choose a Gaussian bunch with $\sigma_{x}=\sigma_{y}=\sigma_{z}=10 \mu \mathrm{m}$ and bending radius $\rho=1 \mathrm{~m}$ at $\gamma=500$ for an illustration of bunch wake.

\section{A. Longitudinal wake}

We numerically compute the wakes in Eq. (29) with various horizontal positions in the bending plane $\zeta=0$. The results are shown in Fig. 5. As we can see, the transverse displacements do not make much difference.

To derive the 1D theory, we start from Eq. (23) for $\Psi_{s}$ and setting $\chi=\zeta=0$ and $\beta=1$,

$$
\Psi_{s}=\frac{e(\cos 2 \alpha-1)}{2 \rho^{2}(\kappa-\sin 2 \alpha)}
$$

Using $\kappa=2 \sin \alpha$, it can be simplified to

$$
\Psi_{s}=\frac{e(\cos 2 \alpha-1)}{4 \rho^{2} \sin \alpha(1-\cos \alpha)} .
$$

By expanding sine and cosine in terms of small $\alpha$, we find,

$$
\Psi_{s} \approx-\frac{e}{\rho^{2} \alpha} .
$$

Moreover, applying the solution of the retarded condition, $\alpha \approx(6 \xi)^{1 / 3}$ for $\xi \geq 0$ with $\xi=\left(z-z^{\prime}\right) /(2 \rho)$, we obtain,

$$
\Psi_{s} \approx-\frac{e}{3^{1 / 3} \rho^{5 / 3}\left(z-z^{\prime}\right)^{1 / 3}},
$$

which leads to the 1D theory,

$$
\mathcal{Y}_{s}\left(z-z^{\prime}\right)=-\frac{2}{3^{1 / 3} \rho^{2 / 3}\left(z-z^{\prime}\right)^{1 / 3}}
$$

and

$$
\delta^{\prime}=\frac{r_{e} N_{b}}{\gamma} \int_{-\infty}^{z} \mathcal{Y}_{s}\left(z-z^{\prime}\right) \frac{\partial \lambda_{b}\left(z^{\prime}\right)}{\partial z^{\prime}} d z^{\prime}
$$

They agree with Eqs. (53) and (54) in Ref. [3]. For a Gaussian bunch, we carry out the integration and derive the bunch wake,

$$
\begin{aligned}
W_{s}(q)= & \frac{2^{11 / 6}}{3^{7 / 3} \rho^{2 / 3} \sigma_{z}^{4 / 3}}\left[\frac{\sqrt{\pi} q_{1} F_{1}\left(\frac{7}{6}, \frac{3}{2},-\frac{q^{2}}{2}\right)}{\sqrt{3} \Gamma\left(\frac{5}{3}\right)}\right. \\
& \left.-\frac{2^{5 / 6} \Gamma\left(\frac{2}{3}\right)_{1} F_{1}\left(\frac{2}{3}, \frac{1}{2},-\frac{q^{2}}{2}\right)}{\Gamma\left(\frac{7}{3}\right)}\right],
\end{aligned}
$$

which is plotted in dashed orange color in Fig. 5. Here $\sigma_{z}$ is the bunch length of the longitudinal distribution and ${ }_{1} F_{1}$ a confluent hypergeometric function of the first kind.

\section{B. Horizontal wake}

We numerically integrate the wakes in Eq. (30) with various horizontal positions in the bending plane and show the results in Fig. 6. The transverse wake is an order of magnitude smaller than the longitudinal one shown in Fig. 5. Moreover, it is largely symmetric with respect to the horizontal displacement of $\chi$, indicating a sextupole wake.

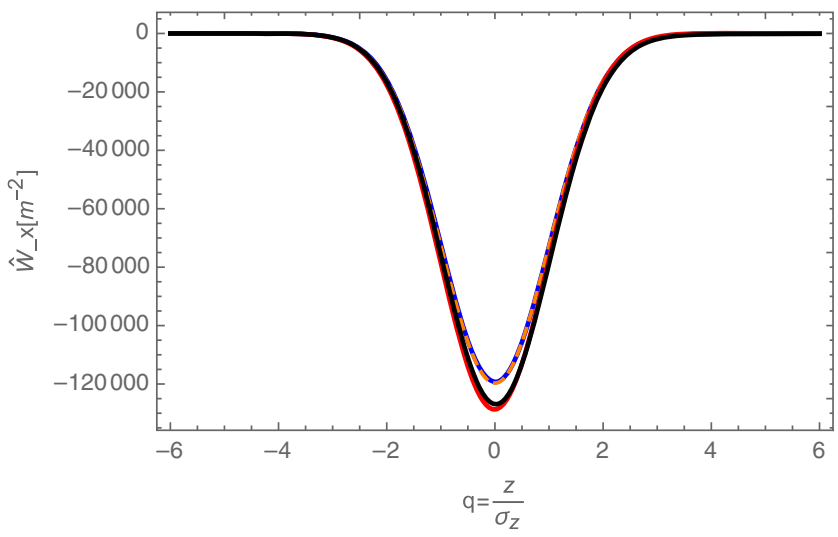

FIG. 6. The horizontal wakes at various horizontal positions: $\chi=-\sigma_{x} / \rho$ (in black color), 0 (blue), and $\sigma_{x} / \rho$ (red) in a comparison to the Gaussian profile (dashed orange) in Eq. (39) with $\Lambda=3$. 


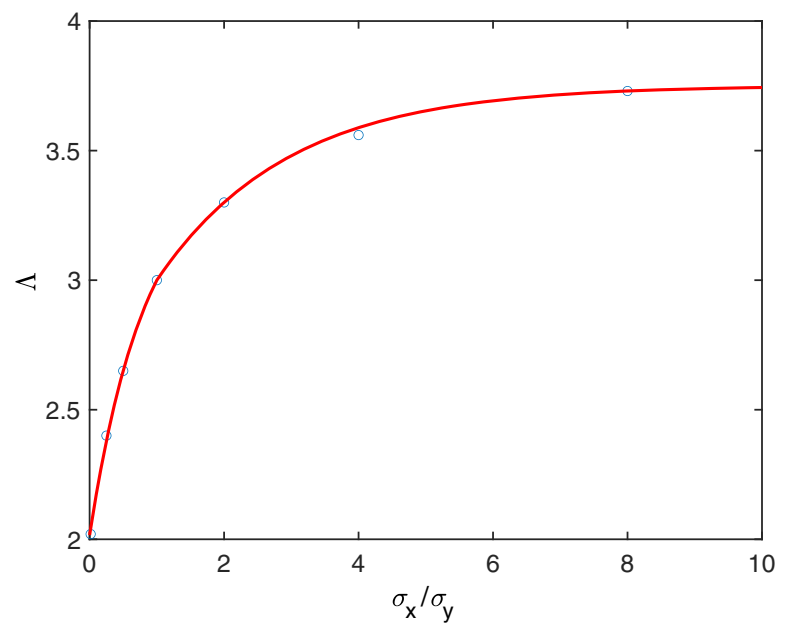

FIG. 7. The coefficient of the residual centripetal force as a function of aspect ratio. The solid line is a fit to exponential curves.

A small asymmetric part can be interpreted as the quadrupole wake. The central wake can be well approximated by a Gaussian profile,

$$
\hat{W}_{x}(q)=-\frac{\Lambda}{\sqrt{2 \pi} \rho \sigma_{z}} \exp \left(-\frac{q^{2}}{2}\right),
$$

as shown in the figure. However, the coefficient is $50 \%$ larger than the value given in Ref. [3].

To understand the difference, we consider a twodimensional theory for a relativistic bunch. Setting $\zeta=0$, $\beta=1$, we find that the terms associated with the incomplete elliptic integrals in $\hat{\Psi}_{x}$ are proportional to $\chi^{2}$. So for small $\chi$, we can approximate,

$\hat{\Psi}_{x} \approx \frac{e\left[\chi(2+\chi)+(1+\chi) \sqrt{\chi^{2}+4(1+\chi) \sin ^{2} \alpha} \sin 2 \alpha\right]}{2 \rho^{2}(1+\chi)[(1+\chi) \cos 2 \alpha-1]}$.

For small $\alpha$, it can be further reduced to,

$$
\hat{\Psi}_{x} \approx \frac{e\left[\chi+\operatorname{sign}(\alpha) 2 \alpha^{2}\right]}{\rho^{2}\left(\chi-2 \alpha^{2}\right)} .
$$

Taking $\chi \rightarrow 0$, we have

$$
\hat{\Psi}_{x}= \begin{cases}-\frac{e}{\rho^{2}}: & \alpha \geq 0 \\ \frac{e}{\rho^{2}}: & \alpha<0,\end{cases}
$$

which leads to,

$$
\hat{\mathcal{Y}}_{x}\left(z-z^{\prime}\right)= \begin{cases}-\frac{2}{\rho}: & z \geq z^{\prime} \\ \frac{2}{\rho}: & z<z^{\prime}\end{cases}
$$

and

$$
x^{\prime \prime}=\frac{r_{e} N_{b}}{\gamma} \int_{-\infty}^{\infty} \hat{\mathcal{Y}}_{x}\left(z-z^{\prime}\right) \frac{\partial \lambda_{b}\left(z^{\prime}\right)}{\partial z^{\prime}} d z^{\prime}
$$

Performing integration by parts and then carrying out the integral, we derive,

$$
x^{\prime \prime}=\frac{-4 r_{e} N_{b}}{\gamma \rho} \lambda_{b}(z)
$$

for the $2 \mathrm{D}$ theory. The residual centrifugal force is a factor of two larger than the formula given by Eq. (38) in Ref. [3], in which half of the radiation from the particles ahead is ignored.

With the understanding of the difference, we continue the numerical evaluation of the transverse wakes with various aspect ratios. We find the coefficient $2 \leq \Lambda \leq 4$ plotted as circles in Fig. 7. The smallest value $\Lambda=2$ is a flat beam in $\mathrm{y}-\mathrm{z}$ plane with zero horizontal size.

\section{Vertical wake}

We numerically calculate the wake in Eq. (31) at the horizontal position of $\chi=0$ and various vertical positions $\zeta$. We find a quadrupole wake that is proportional to the vertical displacements. In particular, we plot the wake at $\zeta=\sigma_{y} / \rho$ in Fig. 8 in comparison to a bunch wake,

$$
\begin{aligned}
W_{y}(q)= & -\frac{\sigma_{y}}{9^{1 / 3} \rho^{4 / 3} \sigma_{z}^{5 / 3}}\left[\frac{\Gamma\left(\frac{2}{3}\right)_{1} F_{1}\left(\frac{5}{6}, \frac{1}{2},-\frac{q^{2}}{2}\right)}{2^{5 / 6} \sqrt{\pi}}\right. \\
& \left.+\frac{2^{5 / 3} \sqrt{\pi} q_{1} F_{1}\left(\frac{4}{3}, \frac{3}{2},-\frac{q^{2}}{2}\right)}{\Gamma\left(-\frac{1}{6}\right)}\right] .
\end{aligned}
$$

Suppressed by $\sigma_{y} / \rho$, the quadrupole wake is very weak in comparison to the horizontal centripetal force. Moreover, the bunch wake can be derived by integrating a Gaussian distribution with the quadrupole wake,

$$
\mathcal{Y}_{y}\left(z-z^{\prime}\right)=-\frac{1}{3^{2 / 3} \rho^{4 / 3}\left(z-z^{\prime}\right)^{2 / 3}},
$$

with

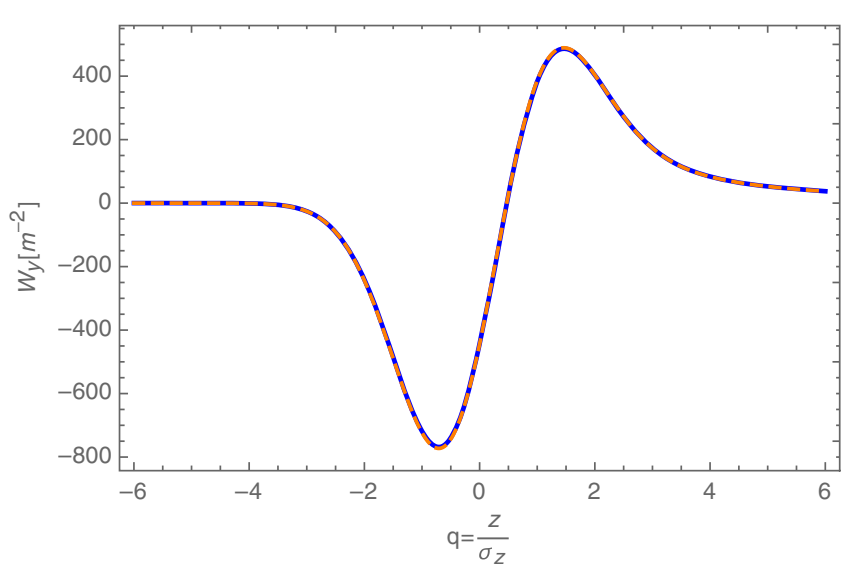

FIG. 8. The vertical wake at a transverse position: $\chi=0$ and $\zeta=\sigma_{y} / \rho$ plotted in blue color. 
TABLE I. Parameters of the last bend in the standard chicane.

\begin{tabular}{lccccccc}
\hline \hline Parameter & $\gamma$ & $\epsilon_{N}$ & $\sigma_{z}$ & $N_{b}$ & $\beta_{x}$ & $\rho$ & $L_{B}$ \\
\hline Value & 9,804 & $1.0 \mu \mathrm{m}$ & $20.0 \mu \mathrm{m}$ & $6.25 \times 10^{9}$ & $5.19 \mathrm{~m}$ & $10.34 \mathrm{~m}$ & $0.5 \mathrm{~m}$ \\
\hline \hline
\end{tabular}

$$
y^{\prime \prime}=\frac{r_{e} N_{b} y}{\gamma} \int_{-\infty}^{z} \mathcal{Y}_{y}\left(z-z^{\prime}\right) \frac{\partial \lambda_{b}\left(z^{\prime}\right)}{\partial z^{\prime}} d z^{\prime},
$$

setting $y=\sigma_{y}$. We shall note that the quadrupole wake in Eqs. (45) and (46) was first obtained by Derbenev and Shiltsev [3].

\section{Estimate of emittance growth}

In a short bending dipole of length $L_{B}$, the increase of normalized emittance due to the longitudinal wake is given by [23],

$$
\Delta \epsilon_{N}^{(s)} \approx 7.5 \times 10^{-3} \frac{\beta_{x}}{\gamma}\left(\frac{r_{e} N_{b} L_{B}^{2}}{\rho^{5 / 3} \sigma_{z}^{4 / 3}}\right)^{2}
$$

where $\beta_{x}$ is the horizontal beta function at the dipole exit where both dispersion and its slope are assumed to be zero. The direct emittance growth due to the centripetal wake can also be estimated using the Gaussian wake in Eq. (39) and written as,

$$
\Delta \epsilon_{N}^{(x)} \approx \frac{(-3+2 \sqrt{3}) \beta_{x}}{24 \pi \gamma}\left(\frac{\Lambda r_{e} N_{b} L_{B}}{\rho \sigma_{z}}\right)^{2} .
$$

Similarly, in the vertical plane, we have,

$$
\Delta \epsilon_{N}^{(y)} \approx \frac{\beta_{y}}{32 \gamma}\left(\frac{r_{e} N_{b} \sigma_{y} L_{B}}{\rho^{4 / 3} \sigma_{z}^{5 / 3}}\right)^{2},
$$

where $\beta_{y}$ is the vertical beta function at the dipole.

For the parameters of the standard chicane [24] in Table I, the estimated emittance growth, indirectly from the energy spread is $10.85 \%$, while the direct contribution from the centripetal wake is $5.32 \%$, assuming a round beam with $\Lambda=3$. In the vertical plane, the growth is about $0.0034 \%$ with $\beta_{y}=12.28 \mathrm{~m}$.

\section{SIMULATION AND MEASUREMENT}

The CSR measurement at the Linac Coherent Light Source (LCLS) was performed and reported in [8]. Here we take the first bunch compressor (BC1) of LCLS as an example to benchmark the theory with the measurement. The layout of the $\mathrm{BC} 1$ section (not scaled) is shown in Fig. 9.

The compressor chicane consists of four identical rectangular bending magnets $\left(\rho=2.172 \mathrm{~m}, L_{B}=0.2034 \mathrm{~m}\right.$, the distance between the first two or the last two dipoles is $2.444 \mathrm{~m}$ and the distance between the middle two is
$0.83 \mathrm{~m}$ ), followed by quadrupole magnets and diagnostic devices such as beam position monitors (BPM), wire scanners and profile monitors. The two central bending magnets in the chicane are motorized so that their horizontal positions can be translated to match the beam trajectory as we change the $\mathrm{R}_{56}$. The incoming beam energy chirp can be controlled by adjusting the rf phase of an upstream linac section (L1S). During the BC1 CSR measurement, the $\mathrm{R}_{56}$ was fixed at $-45.5 \mathrm{~mm}$, and we only adjusted the L1S phase for changing the beam energy chirp. The beam energy at $\mathrm{BC} 1$ was held constant at $250 \mathrm{MeV}$ through an energy feedback control of the L1S amplitude. The L1X section, a fourth-harmonic linearizer, was set at constant voltage and phase (20 MV, $-160 \mathrm{deg}$ ).

As we move the L1S phase off the crest of the sinusoidal curve, the beam chirp is getting larger, resulting in more compression of the bunch. (This is called an undercompression mode) until achieving a minimum of the bunch length (full-compression mode). After the full compression point, further increasing the beam chirp will cause a swap of the bunch head and tail after passing through the chicane, and the bunch becomes getting longer (overcompression mode). At each point of this L1S phase, we measured the beam emittance with the quad scan method using the downstream wire scanners, as well as the centroid beam positions with the three downstream BPMs. The incoming beam trajectory before the chicane was maintained at the center of the BPMs in the horizontal plane.

To simulate the bunch compression through the $\mathrm{BC}$, we performed start-to-end simulation from the photocathode gun. The injector section up to $135 \mathrm{MeV}$ was simulated with a 3-D space charge code IMPACT [25]. Then the particles are dumped to ELEGANT code [26], tracking through L1S, L1X, and the BC1 chicane section. A 1-D CSR model with transient effect is included in the ELEGANT simulation. Downstream from the chicane, the simulated beam emittance and centroid position at the three BPM

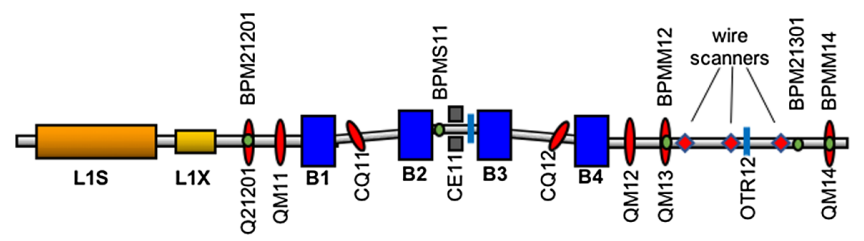

FIG. 9. A layout of the first bunch compressor $\mathrm{BC} 1$ area at the LCLS. The wire scanners are used for emittance measurement after compression, and the downstream BPMs (BPMM12, BPM21301 and BPMM14) are used to measure the beam centroid transverse position. 

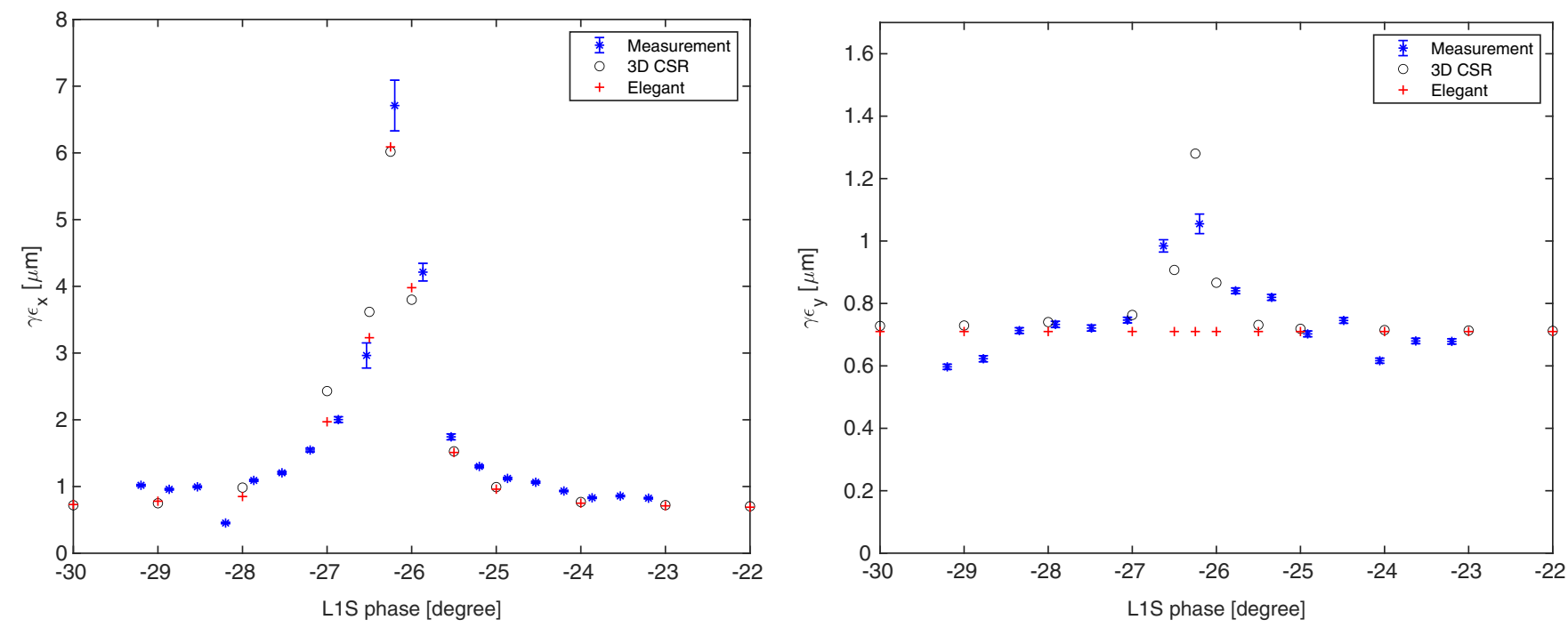

FIG. 10. A comparison of the measurements and simulations of the horizontal (on left) and vertical (on right) emittance for the first bunch compressor in the LCLS.
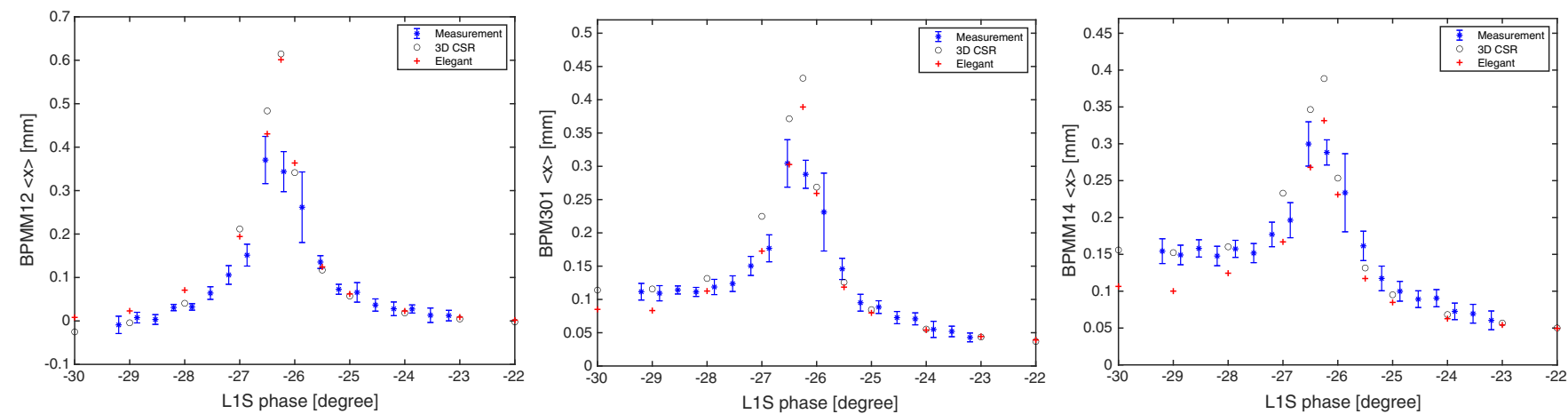

FIG. 11. A comparison of the measurements and simulations of the horizontal beam position for the first bunch compressor in the LCLS.

locations are plotted in Figs. 10 and 11, together with measurement data. At each L1S phase configuration during the ELEGANT run, we also saved the particle distribution at the entrance of the chicane, which will be used for the 3-D CSR studies developed in this paper.

The main beam parameters of the dumped particle from the ELEGANT simulation at the chicane entrance are listed in

TABLE II. Beam parameters at the entry of the BC1 in LCLS.

\begin{tabular}{ll}
\hline \hline Parameter & \multicolumn{1}{c}{ Value } \\
\hline Beam energy, $E_{0}[\mathrm{GeV}]$ & 0.25 \\
Bunch charge, $C[\mathrm{pC}]$ & 250 \\
Normalized emittance, $\epsilon_{N}^{(x)}, \epsilon_{N}^{(y)},[\mu \mathrm{m}]$ & $0.7,0.7$ \\
Slice energy spread, $\sigma_{E} / E_{0}$ & $5 \times 10^{-5}$ \\
Bunch length, $\sigma_{z}[\mathrm{~mm}]$ & 0.75 \\
Beta function, $\beta_{x}, \beta_{y}[\mathrm{~m}]$ & $24.65,3.43$ \\
Alpha function, $\alpha_{x}, \alpha_{y}$ & $3.64,1.46$ \\
\hline \hline
\end{tabular}

Table II. The energy chirp $h$ varies from about 19 to $24 \mathrm{~m}^{-1}$ depending on the L1S phase. It is well known that the steady-state wake in Eq. (37) is not adequate for the short bending magnets $\left(L_{B} \leq\left(24 \sigma_{z} \rho^{2}\right)^{1 / 3}\right)$ in the bunch compressor. Instead, the transient wakes [5,6] should be applied. So for our 3D CSR simulations, we started with simpler transient wakes [27] in the longitudinal plane both in the bends and their down-stream drifts. The code was checked against the ELEGANT simulations with excellent agreements. We then added the centripetal force in Eq. (43) and the quadrupole wake in Eq. (46) in the horizontal and vertical planes respectively in the bends.

The particles through the chicane applied the CSR wakefields as an additional kick in a hundred slices of each bend and drift. The wakes are computed in a longitudinal mesh with 3200 grid points. Here we deployed a method that is similar to the convolution of the wake with the longitudinal bunch distribution in the study of the microwave instability in electron storage rings [28]. 
Moreover, to accurately model a round beam, we also replaced the coefficient 4 with $\Lambda=1.5$ in Eq. (43) for the horizontal kick. The smaller number is considered as an empirical and effective value that may be justified by the effects of ramping up in the transient region. The theory including the transient is under investigation.

The simulation results of the emittance growth due to 3-D CSR wakes as the bunch goes through a maximal compression are also shown in Fig. 10. We see both the 3-D CSR simulations and the 1-D CSR results from ELEGANT agree well with the measured horizontal emittance. There is not much growth in the horizontal emittance due to the centripetal force. In the vertical plane, there is no emittance effect from the 1-D CSR model, while the 3-D CSR model gives an emittance growth at the strong compression region, which is consistent with measurement. The agreement could be better if we adjust the initial emittance. The result shows that the quadrupole wake is the main source of the growth of the vertical emittance.

For a consistency check, we also plotted the horizontal beam position at the three down-stream BPM locations in Fig. 11. In general we see good agreement between the 1-D, 3-D CSR and the measurement. Note that we have shifted the readings of the BPM by a constant assuming a static offset of the measured BPM position. At the last BPM (BPMM14), we see that at the over-compression side the 1-D CSR model underestimates the BPM offset, while the 3-D CSR model agrees better with the measured data. This could be explained by the centripetal force. The positive readings are corresponding with the upward direction in Fig. 9. The direction is consistent to the energy loss and the kick by the centripetal force in the last bend. The result confirms the cancellation of the centrifugal force resulting in a residual centripetal force.

\section{SUMMARY}

We have developed a three dimensional theory for steady-state CSR in free space. The theory resolves the retardation effort and reduces the dynamical effect to the three dimensional integrals of convolution with the longitudinal derivative of the instantaneous bunch distribution. The kernels are written in terms of analytical functions, including the incomplete elliptic integrals. Moreover, it is self-consistent with a proper treatment of the singularity and ready to be incorporated in a computer program for accurate simulation of three dimensional effects. The critical work will be to speed up the integrations, perhaps utilizing parallel computing.

Most importantly, we essentially rederive the results found by Derbenev and Shiltsev with a modification of the coefficient of the centripetal force in the ultrarelativistic limit. For the round beam, the coefficient $\Lambda=3$ is $50 \%$ larger than the previous value. The measurement in the first bunch compressor of LCLS supports our finding only if the transient effects are considered. Nevertheless, we can conclude that indeed there is a cancellation of the centrifugal force but left with a residual centripetal force. We therefore resolve the historical controversy from a viewpoint of the theory and the measurement.

As a by-product, we have developed a simplified threedimensional model based on the wakefields. It has captured the most important aspects of the CSR, namely the wellknown longitudinal wake with transient, the centripetal force in the horizontal plane, and the quadrupole wake in the vertical plane. It can be considered as another important step toward developing a robust computer program for simulating the three dimensional effects of CSR.

Our 3D theory is applicable if the shielding due to metallic boundary can be neglected. The effect can be characterized by the condition on the shielding parameter: $\sigma_{z} \rho^{1 / 2} / h^{3 / 2} \ll 1$, where $h$ is the distance between two parallel metal planes. The condition is satisfied in typical bunch compressors where $\sigma_{z} \ll 1 \mathrm{~mm}, \rho \sim \mathrm{m}$, and $h \sim \mathrm{cm}$. Moreover, our steady-state theory is valid only if the characteristic overtaking length: $\left(24 \sigma_{z} \rho^{2}\right)^{1 / 3}$ is less than $L_{B}$ the dipole length. For some compressors, this condition is not satisfied and the transient effects should be properly taken into account. Developing a 3D theory that includes the transient effects is in good progress.

\section{ACKNOWLEDGMENTS}

We would like to thank Karl Bane, Alexander Chao, Zhirong Huang, Kazuhito Ohmi, and Gennady Stupakov for many helpful discussions. This work was supported by the Department of Energy under Contract No. DE-AC0276 SF00515.

\section{APPENDIX A: SOLUTION OF THE QUARTIC EQUATION}

In general, we would like to find the roots of the depressed quartic equation:

$$
\alpha^{4}+v \alpha^{2}+\eta \alpha+\zeta=0 .
$$

It has an analytical solution discovered by Ferrari (1522-1565). The method finds a value $m$ to factorize the fourth-order polynomial by a product of two quadratic polynomials. It turns out that $\mathrm{m}$ itself is a solution of a third-order equation and is given by,

$$
m=-\frac{v}{3}+\left(\frac{\zeta}{3}+\frac{v^{2}}{36}\right) \Omega^{-1 / 3}+\Omega^{1 / 3},
$$

where

$$
\Omega=\frac{\eta^{2}}{16}-\frac{\zeta v}{6}+\frac{v^{3}}{216}+\sqrt{\left(\frac{\eta^{2}}{16}-\frac{\zeta v}{6}+\frac{v^{3}}{216}\right)^{2}-\left(\frac{\zeta}{3}+\frac{v^{2}}{36}\right)^{3}} .
$$



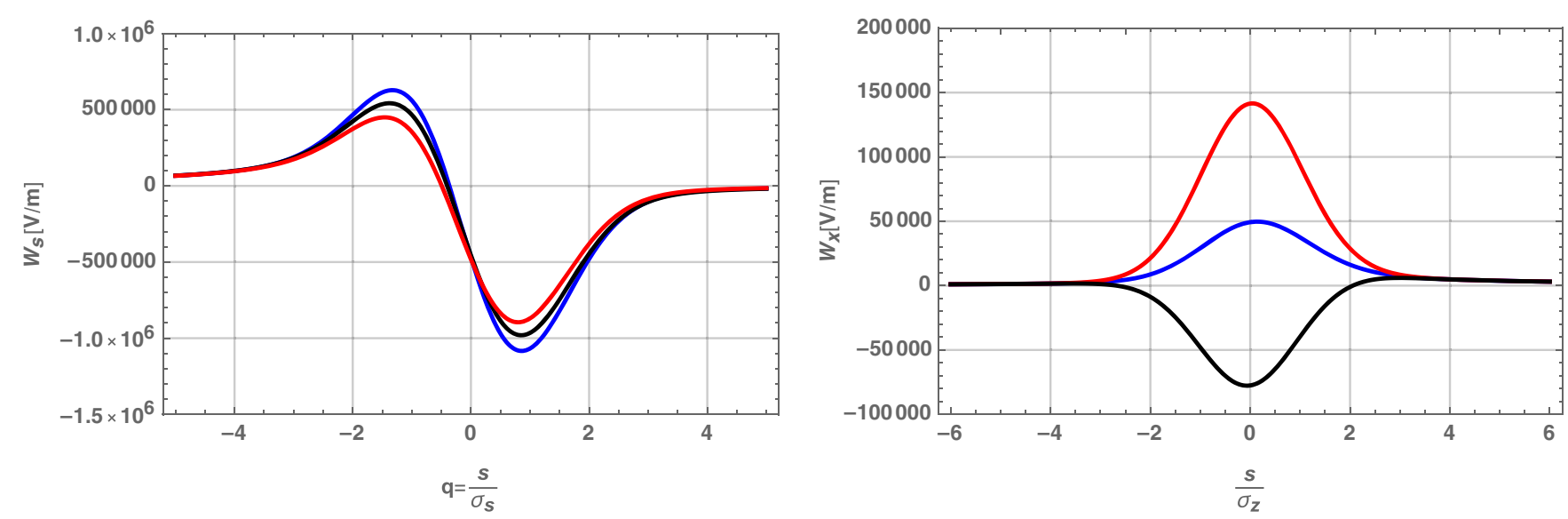

FIG. 12. Longitudinal (left) and horizontal (right) forces at various of horizontal offsets, $\delta x=2.5 \sigma$ (red), 0 (blue), $-2.5 \sigma$ (black), for a Gaussian bunch moving along a circle with radius, $\rho=10 \mathrm{~m}$ at energy of $\gamma=100$.

Then we have,

$\alpha= \begin{cases}\frac{1}{2}\left(\sqrt{2 m}+\sqrt{-2(m+v)-\frac{2 \eta}{\sqrt{2 m}}}\right) & \text { if } \xi \geq 0 \\ \frac{1}{2}\left(-\sqrt{2 m}+\sqrt{-2(m+v)+\frac{2 \eta}{\sqrt{2 m}}}\right) & \text { if } \xi<0 .\end{cases}$

These are two real roots of the quartic equation. They cover two different regions: ahead or behind the source particle.

\section{APPENDIX B: SPACE CHARGE}

The first term or the nonradiative electromagnetic fields in the Lienard-Wiechert formula can also be computed and their components are given by,

$$
E_{x}^{(s c)}=\frac{e[(1+\chi)-\cos 2 \alpha-\beta \kappa \sin 2 \alpha)]}{\gamma^{2} \rho^{2}[\kappa-\beta(1+\chi) \sin 2 \alpha]^{3}}
$$

$$
\begin{aligned}
E_{y}^{(s c)} & =\frac{e \zeta}{\gamma^{2} \rho^{2}[\kappa-\beta(1+\chi) \sin 2 \alpha]^{3}}, \\
E_{s}^{(s c)} & =\frac{e(\sin 2 \alpha-\beta \kappa \cos 2 \alpha)}{\gamma^{2} \rho^{2}[\kappa-\beta(1+\chi) \sin 2 \alpha]^{3}}, \\
B_{x}^{(s c)} & =-\frac{e \beta \zeta \cos 2 \alpha}{\gamma^{2} \rho^{2}[\kappa-\beta(1+\chi) \sin 2 \alpha]^{3}}, \\
B_{y}^{(s c)} & =\frac{e \beta[(1+\chi) \cos 2 \alpha-1]}{\gamma^{2} \rho^{2}[\kappa-\beta(1+\chi) \sin 2 \alpha]^{3}}, \\
B_{s}^{(s c)} & =\frac{e \beta \zeta \sin 2 \alpha}{\gamma^{2} \rho^{2}[\kappa-\beta(1+\chi) \sin 2 \alpha]^{3}} .
\end{aligned}
$$

Since they are all are suppressed by $\gamma^{2}$, they become important only at low energy. Similar to CSR, we can introduce their potentials as,

$$
\begin{aligned}
& \Psi_{x}^{(s c)}(\chi, \zeta, \xi)=\frac{e}{2 \gamma^{2} \rho^{2}}\left\{\frac{F\left(\alpha, \frac{-4(1+\chi)}{\chi^{2}+\zeta^{2}}\right)}{(1+\chi) \sqrt{\chi^{2}+\zeta^{2}}}+\frac{\left[\chi(2+\chi)-\zeta^{2}\right] E\left(\alpha, \frac{-4(1+\chi)}{\chi^{2}+\zeta^{2}}\right)}{(1+\chi)\left[\zeta^{2}+(2+\chi)^{2}\right] \sqrt{\chi^{2}+\zeta^{2}}}+\frac{\beta(\cos 2 \alpha-1-\chi)}{\left[\kappa^{2}-\beta^{2}(1+\chi)^{2} \sin ^{2} 2 \alpha\right]}\right. \\
& -\frac{\kappa\left[\chi(2+\chi)\left(\beta^{2}(1+\chi)^{2}-2\right)+\zeta^{2}\left(2+\beta^{2}(1+\chi)^{2}\right)\right] \sin 2 \alpha}{\left[\zeta^{4}+\chi^{2}(2+\chi)^{2}+2 \zeta^{2}\left(2+2 \chi+\chi^{2}\right)\right]\left[\kappa^{2}-\beta^{2}(1+\chi)^{2} \sin ^{2} 2 \alpha\right]} \\
& \left.-\frac{\kappa \beta^{2}(1+\chi)\left[\chi(2+\chi)-\zeta^{2}\right] \sin 2 \alpha \cos 2 \alpha}{\left[\zeta^{4}+\chi^{2}(2+\chi)^{2}+2 \zeta^{2}\left(2+2 \chi+\chi^{2}\right)\right]\left[\kappa^{2}-\beta^{2}(1+\chi)^{2} \sin ^{2} 2 \alpha\right]}\right\} \\
& \Psi_{y}^{(s c)}(\chi, \zeta, \xi)=\frac{e \zeta}{2 \gamma^{2} \rho^{2}}\left\{\frac{2 E\left(\alpha, \frac{-4(1+\chi)}{\chi^{2}+\zeta^{2}}\right)}{\left[\zeta^{2}+(2+\chi)^{2}\right] \sqrt{\chi^{2}+\zeta^{2}}}-\frac{\beta}{\left[\kappa^{2}-\beta^{2}(1+\chi)^{2} \sin ^{2} 2 \alpha\right]}\right. \\
& +\frac{\kappa(1+\chi)\left[\beta^{2}\left(2+2 \chi+\chi^{2}+\zeta^{2}\right)-4+2 \beta^{2}(1+\chi) \cos 2 \alpha\right] \sin 2 \alpha}{\left[\zeta^{4}+\chi^{2}(2+\chi)^{2}+2 \zeta^{2}\left(2+2 \chi+\chi^{2}\right)\right]\left[\kappa^{2}-\beta^{2}(1+\chi)^{2} \sin ^{2} 2 \alpha\right]}, \\
& \Psi_{s}^{(s c)}(\chi, \zeta, \xi)=-\frac{e}{2 \gamma^{2} \rho^{2}(1+\chi)[\kappa-\beta(1+\chi) \sin 2 \alpha]},
\end{aligned}
$$


with $\mathcal{Y}_{s}^{(s c)}=2 \rho \Psi_{s}^{(s c)} / e, \mathcal{Y}_{x}^{(s c)}=2 \rho \Psi_{x}^{(s c)} / e$, and $\mathcal{Y}_{y}^{(s c)}=$ $2 \rho \Psi_{y}^{(s c)} / e$ as the kernels for computing the bunch wakes. As an example, we compute numerically the longitudinal and horizontal wakes of a spherical and Gaussian bunch with $\sigma=50 \mu \mathrm{m}$, including both CSR and space charge contributions but without $\Psi_{\phi}$ at $\gamma=100$. The wakes are translated to the forces for $1 \mathrm{nC}$ bunch charge in MKS unit shown in Fig. 12 for a comparison to the previously known results by Dohlus, Kabel, and Limberg [29]. In addition, the longitudinal direction is also reversed to comply with their convention. The agreement is excellent.

[1] S. Heifets, G. Stupakov, and S. Krinsky, Coherent synchrotron radiation instability in a bunch compressor, Phys. Rev. Accel. Beams 5, 064401 (2002).

[2] Z. Huang and K-J. Kim, Formulas for coherent synchrotron radiation microbunching in a bunch compressor chicane, Phys. Rev. Accel. Beams 5, 074401 (2002).

[3] Y.S. Derbenev, J. Rossbach, E. L. Saldin, and V. D. Shiltsev, Microbunch radiative tail-head interaction, DESY Print Report No. TESLA-FEL 95-05, Hamburg, 1995.

[4] J. B. Murphy, S. Krinsky, and R. L. Gluckstern, Longitudinal wakefield for an electron moving on a circular orbit, Part. Accel. 57, 9 (1997).

[5] M. Dohlus and T. Limberg, Emittance growth due to wake fields on curved bunch trajectories, Nucl. Instrum. Methods Phys. Res., Sect. A 393, 494 (1997).

[6] E. L. Saldin, E. A. Schneidmiller, and M. V. Yurkov, On the coherent radiation of an electron bunch moving in arc of a circle, Nucl. Instrum. Methods Phys. Res., Sect. A 398, 373 (1997).

[7] M. Borland, Simple method for particle tracking with coherent synchrotron radiation, Phys. Rev. Accel. Beams 4, 070701 (2001).

[8] K. L.F. Bane et al., Measurements and modeling of coherent synchrotron radiation and its impact on the Linac Coherent Light Source electron beam, Phys. Rev. Accel. Beams 12, 030704 (2009).

[9] R. Talman, Novel Relativistic Effect Important in Accelerator, Phys. Rev. Lett. 56, 1429 (1986).

[10] E. P. Lee, Cancellation of the centrifugal space-charge force, Part. Accel. 25, 241 (1990).

[11] Y.S. Derbenev and V.D. Shiltsev, Transverse effects of microbunch radiative interaction, SLAC National Accelerator Laboratory Report No. SLAC-PUB-7181, 1996.

[12] G. V. Stupakov, Centripetal transverse wakefield in relativistic beam, arXiv:1901.10745v2.
[13] R. Li and Y. Derbenev, Discussions of the cancellation effect on a circular orbit, Jefferson Lab Report No. JLABTN-02-054, 2003.

[14] G. Geloni, E. Saldin, E. Schneidmiller, and M. Yurkov, Misconceptions regarding the cancellation of self-forces in the transverse equation of motion for an electron in a bunch, arXiv:physics/0310133v1.

[15] D. Gillingham and T. Antonsen, Calculation of coherent synchrotron radiation in toroidal waveguides by paraxial wave equation, Phys. Rev. Accel. Beams 10, 054402 (2007).

[16] A. Novokhatski, Field dynamics of coherent synchrotron radiation using a direct numerical solution of Maxwell equations, Phys. Rev. Accel. Beams 14, 060707 (2011).

[17] R. Li, Curvature-induced bunch self-interaction for an energy-chirped bunch in magnetic bends, Phys. Rev. Accel. Beams 11, 024401 (2008).

[18] G. Bassi, J. Ellison, K. Heineman, and R. Warnock, Microbunching instability in a chicane: Two-dimensional mean field treatment, Phys. Rev. Accel. Beams 12, 080704 (2009).

[19] M. Dohlus and T. Limberg, CSRtrack: Faster calculation of 3-D CSR effects, in Proceedings of FEL2004 Conference (Comitato Conferenze Elettra, Trieste, Italy, 2004), pp. 18-21.

[20] Y. Cai, Coherent synchrotron radiation by electrons moving on circular orbits, Phys. Rev. Accel. Beams 20, 064402 (2017).

[21] J. D. Jackson, Classical Electrodynamics, 3rd ed. (John Wiley \& Son, New York, 1999).

[22] E. D. Courant and H. S. Snyder, Theory of the alternatinggradient synchrotron, Ann. Phys. (Berlin) 3, 1 (1958).

[23] G. V. Stupakov, Effect of centrifugal transverse wakefield for microbunch in bend, SLAC National Accelerator Laboratory Report No. SLAC-PUB-8028, 2006.

[24] L. Giannessi, Simulation codes for high brightness electron beam free-electron laser experiments, Phys. Rev. Accel. Beams 6, 114802 (2003).

[25] J. Qiang, S. Lidia, R. D. Ryne, and C. Limborg-Deprey, Three-dimensional quasistatic model for high brightness beam dynamics simulation, Phys. Rev. Accel. Beams 9, 044204 (2006).

[26] M. Borland, Elegant, Argonne National Laboratory Advanced Photon Source Report No. LS-287, 2000.

[27] G. Stupakov and P. Emma, CSR wake for a short magnet in ultrarelativistic limit, SLAC National Accelerator Laboratory Report No. SLAC-PUB-9242, 2002.

[28] K. L. F. Bane, Y. Cai, and G. Stupakov, Threshold studies of the microwave instability in electron storage rings, Phys. Rev. Accel. Beams 13, 104402 (2010).

[29] M. Dohlus, A. Kabel, and T. Limberg, Efficient field calculation of 3D bunches on general trajectories, Nucl. Instrum. Methods Phys. Res., Sect. A 445, 338 (2000). 